\title{
Reviewers in this Issue
}

Prof. A. M. M. Shariful Alam

Department of Clinical Oncology

Enam Medical College \& Hospital, Savar, Dhaka

Prof. Mahmuda Begum

Department of Pharmacology \& Therapeutics

Enam Medical College, Savar, Dhaka

Prof. Gulshan Ara

Department of Obstetics \& Gynaecology

Enam Medical College \& Hospital, Savar, Dhaka

Prof. Md. Rezwanur Rahman

Department of Biochemistry

Delta Medical College, Dhaka

Prof. Irin Perveen

Department of Gastroenterology

Enam Medical College \& Hospital, Savar, Dhaka

Dr. Md. Khalilur Rahman

Associate Professor

Department of Radiology \& Imaging

Dhaka Medical College \& Hospital, Dhaka

Dr. Mithun Alamgir

Associate Professor

Department of Community Medicine

Enam Medical College, Savar, Dhaka

Dr. Rukhsana Parvin

Associate Professor

Department of Medicine

Enam Medical College \& Hospital, Savar, Dhaka

Dr. Ishrat Jahan

Associate Professor

Department of Paediatrics

Enam Medical College \& Hospital, Savar, Dhaka 